
\title{
$\begin{array}{ll}\text { Research Square } & \text { They should not be considered conclusive, used to inform clinical practice, } \\ \text { or referenced by the media as validated information. }\end{array}$ \\ Fifth fundamental force and formulation to determine it
}

Raghvendra Singh ( $\square$ raghvend@iitk.ac.in )

Indian Institute of Technology Kanpur

Physical Sciences - Article

Keywords: forces of nature, fifth force, surface tension, quantum vacuum field

Posted Date: July 19th, 2021

DOI: https://doi.org/10.21203/rs.3.rs-729130/v1

License: (c) (i) This work is licensed under a Creative Commons Attribution 4.0 International License.

Read Full License 


\section{Fifth fundamental force and formulation to determine it}

Raghvendra Singh*

Department of Chemical Engineering, Indian Institute of Technology Kanpur, India

*Correspondence to:

Raghvendra Singh, Ph.D.

Assistant Professor

Department of Chemical Engineering

Indian Institute of Technology Kanpur

Kanpur, India, 208016

Tel: 91-512-259 7605

Fax: 91-512-259 0104

Email: raghvend@iitk.ac.in 


\begin{abstract}
There are four known fundamental forces of nature and there is a need to combine them into a unified theory. Progress has been made toward this goal but gravity remains an issue. However, the four forces are body forces that act on points. They together do not make the universe a closed system. Here, I identify a surface force, which acts outward normal to the surface of the universe. Further, using water drop hanging in a vacuum as a model, I provide a formula to find the magnitude of this force. The fifth force may be generated by surface tension, a property of the quantum vacuum field.
\end{abstract}




\section{Introduction}

In the early universe, matter may have frozen out due to quantum fluctuation ${ }^{1,2}$ of a quantum vacuum field ${ }^{3}$. Further, classicalization of the quantum fluctuation may be responsible for the structure formation in the early universe ${ }^{4,5}$. Furthermore, the freeze-out may be responsible for the expansion of the universe, and the origin of galaxies has been explained based on a first-order phase transition of the vacuum ${ }^{6}$.

Since mass at the surface of the universe is pulled inward by the gravitational field, one can conclude that in absence of an opposite surface force, the surface of the universe will be decelerating. Since the rest of the universe is expanding at equilibrium, the deceleration of the surface will make the universe an open system. Therefore, I postulate that a fifth fundamental force, acting outward normal to the surface of the universe, exists.

To find the surface force, I consider the universe analogous to a water drop hanging in a vacuum or a medium. An inward normal force acts on the surface of the water drop because of an imbalance between the higher cohesive force between the surface and interior water molecules and the lower adhesive force between surface water molecules and outside medium (or vacuum). This compressive force is balanced by the surface tension of the water drop. The net result of the surface tension is an outward normal force, acting on the surface of the drop, balancing the difference between the cohesive and adhesive forces.

Here, I present a surface tension model of the fifth force. Using water drop as a model, I provide a formula to determine the magnitude of this force. Further, I compare this formula with the one derived by considering that the surface tension force originates from the quantum vacuum field. 


\section{Results}

\section{Formulation to determine the fifth fundamental force}

One of the cohesive/adhesive forces acting on the surface of the universe, $F_{c g}$, can be given by Newton's law of gravitation as:

$F_{c g}=-G \frac{m_{i} m_{s}}{r^{2}}$

where, $m_{i}$ is the internal mass of the universe, $m_{s}$ is the mass at the surface of the universe, and $\mathrm{r}$ is its radius.

Other cohesive and adhesive forces acting on the surface are the electromagnetic force, the strong force, and the weak force. Since Newton's second law of motion, applies to an inertial frame lacking acceleration, the universe can be assumed to be expanding at equilibrium. Thus, the net force, $\mathrm{F}$, acting in the normal direction on the surface:

$F=\sum_{i} F_{c i}+F_{s}=0$

where, $F_{c i}$ is a cohesive/adhesive force acting on the surface and $\mathrm{F}_{\mathrm{s}}$ is the surface tension force that must act outward normal on the surface.

Based on the analogy of the universe with a water drop hanging in a vacuum, the force $\mathrm{F}_{\mathrm{s}}$ can be given by the Young-Laplace equation.

$F_{s}=\frac{2 \gamma}{r} \cdot 4 \pi r^{2}$

where, $\gamma$ is the surface tension of the universe.

Further, besides the Young-Laplace equation, the surface tension force can also be determined from the quantum vacuum field. The derivation of Ford ${ }^{3}$, which is devised to remove divergences of the energy-momentum tensor by introducing a cut-off function, to determine the energy density and pressure of the quantum vacuum field is being reproduced here with a difference that, here, I choose a different cut-off function to account for a variable field strength while Ford chose a constant field strength of unity.

The field strength $\mathrm{T}$ is defined based on the density of the field and volume of the universe: 
$T=\left(\frac{4}{3} \pi r^{3}\right) \rho_{v a c}$

The cut-off function $\mathrm{f}$ is chosen to remove the ultraviolet divergences when the wave number $k \rightarrow \infty$. Thus, when $k \rightarrow \infty, f \rightarrow 0$. Further, for long-wavelength modes, when $k \rightarrow 0, f \rightarrow$ T. A suitable choice of, $\mathrm{f}$, is:

$f=\left(\frac{4}{3} \pi r^{3}\right) \rho_{v a c} e^{-\alpha k}$

The field satisfies the equation ${ }^{3}$ :

$\square \psi+\frac{1}{6} R \psi+\mu^{2} \psi=0$

Where, $\square=\nabla_{\alpha} \nabla^{\alpha}$, R is the scalar curvature, and $\mu$ is the mass of the field. For the conformal scalar field, the energy-momentum tensor, $T_{\alpha \beta}$ is ${ }^{3}$ :

$T_{\alpha \beta}=\psi{ }_{, \alpha} \psi_{, \beta}-\frac{1}{2} g_{\alpha \beta} \psi_{{ }_{\rho} \rho} \psi^{\prime} \rho-\frac{1}{6} \nabla_{\alpha}\left(\psi^{2}\right)_{{ }_{\beta}}+\frac{1}{6} g_{\alpha \beta} \square\left(\psi^{2}\right)-\frac{1}{6} G_{\alpha \beta} \psi^{2}+\frac{1}{2} g_{\alpha \beta} \mu^{2} \psi^{2}$

Where, $G_{\alpha \beta}$ is the Einstein tensor. The vacuum expectation value of the energy-momentum tensor is ${ }^{3}$ :

$\rho_{0}=\frac{1}{4 \pi^{2} r^{3}} \sum_{n=0}^{\infty}(n+1)^{2} \omega_{n}$

$P_{0}=\frac{1}{4 \pi^{2} r^{3}} \sum_{n=0}^{\infty}(n+1)^{2}\left(\frac{\omega_{n}^{2}-\mu^{2}}{3 \omega_{n}}\right)$

where, $\omega_{n}=\frac{\left\{(n+1)^{2}+\mu^{2} r^{2}\right\}^{1 / 2}}{r}$ are the eigenfrequencies with a degeneracy of $(n+1)^{2} \cdot \rho_{0}$ is the energy density and $P_{0}$ is the pressure. $\rho_{0}=\left\langle 0\left|T_{0}^{0}\right| 0\right\rangle$ and $P_{0}=-\left\langle 0\left|T_{1}^{1}\right| 0\right\rangle=-\left\langle 0\left|T_{2}^{2}\right| 0\right\rangle=$ $-\left\langle 0\left|T_{3}^{3}\right| 0\right\rangle$. These are the divergent quantities. The cut-off dependent quantities $\tilde{\rho}$ and $\widetilde{P}$ are determined from equations (8) and (9) as:

$\tilde{\rho}=\frac{1}{4 \pi^{2} r^{3}}\left\{\left(\frac{4}{3} \pi r^{3}\right) \rho_{v a c}\right\} \sum_{m=1}^{\infty} m^{2}\left(k_{m}^{2}+\mu^{2}\right)^{1 / 2} e^{-\alpha k_{m}}$
$\tilde{P}=\frac{1}{12 \pi^{2} r^{3}}\left\{\left(\frac{4}{3} \pi r^{3}\right) \rho_{v a c}\right\} \sum_{m=1}^{\infty} m^{4}\left(k_{m}^{2}+\mu^{2}\right)^{-1 / 2} e^{-\alpha k_{m}}$

where, $k_{m}=\frac{m}{r}$ 
Substractions $\bar{\rho}$ and $\bar{P}$ are calculated by letting $r \rightarrow \infty$. From equations 10 and 11 ,

$$
\begin{aligned}
& \bar{\rho}=\lim _{r \rightarrow \infty} \tilde{\rho}=\frac{1}{4 \pi^{2}}\left\{\left(\frac{4}{3} \pi r^{3}\right) \rho_{v a c}\right\} \int_{0}^{\infty} k^{2}\left(k^{2}+\mu^{2}\right)^{1 / 2} e^{-\alpha k_{m}} \mathrm{dk} \\
& \bar{P}=\lim _{r \rightarrow \infty} \tilde{P}=\frac{1}{12 \pi^{2}}\left\{\left(\frac{4}{3} \pi r^{3}\right) \rho_{v a c}\right\} \int_{0}^{\infty} k^{4}\left(k^{2}+\mu^{2}\right)^{-1 / 2} e^{-\alpha k_{m}} d k
\end{aligned}
$$

These quantities are the eigenvalues of the energy-momentum tensor when $t \rightarrow \infty$ for an expanding universe ${ }^{3}$. Then the physical energy density and pressure in the Einstein universe $\operatorname{are}^{3}$ :

$\rho=\lim _{\alpha \rightarrow 0}(\tilde{\rho}-\bar{\rho})$

$P=\lim _{\alpha \rightarrow 0}(\tilde{P}-\bar{P})$

Following the rest of the derivation by Ford ${ }^{3}$,

$\rho=\frac{1}{4 \pi^{2} r^{4}}\left\{\left(\frac{4}{3} \pi r^{3}\right) \rho_{v a c}\right\}\left\{\frac{1}{120}-\frac{1}{24} \tau^{2}-\frac{1}{8} \tau^{4}\left(\ln \frac{\tau}{2}+e+\frac{1}{4}\right)+\sum_{m=1}^{M-1}\left[m^{2}\left(m^{2}+\tau^{2}\right)^{\frac{1}{2}}-m^{3}-\right.\right.$

$\left.\left.\frac{1}{2} \tau^{2} m+\frac{\tau^{4}}{8 m}\right]+\sum_{n=3}^{\infty} b_{n} \tau^{2 n} \sum_{m=M}^{\infty} m^{3-2 n}\right\}$

and

$P=\frac{1}{12 \pi^{2} r^{4}}\left\{\left(\frac{4}{3} \pi r^{3}\right) \rho_{v a c}\right\}\left\{\frac{1}{120}+\frac{1}{24} \tau^{2}+\frac{1}{8} \tau^{4}\left(3 \ln \frac{\tau}{2}+3 e+\frac{7}{4}\right)+\sum_{m=1}^{M-1}\left[m^{4}\left(m^{2}+\tau^{2}\right)^{-\frac{1}{2}}-\right.\right.$

$\left.\left.m^{3}+\frac{1}{2} \tau^{2} m-\frac{3 \tau^{4}}{8 m}\right]+\sum_{n=3}^{\infty} c_{n} \tau^{2 n} \sum_{m=M}^{\infty} m^{3-2 n}\right\}$

Where,

$\tau=\mu r$ and $\mathrm{M}$ is any integer greater than $\tau$.

$b_{n}=(-1)^{n-1}(2 n-3) ! ! / 2^{n} n !$

$c_{n}=(-1)^{n-1}(2 n-1) ! ! / 2^{n} n !$

and $e=0.577$...is the Euler's constant.

Thus, from equations 16 and 17, for a massless $(\mu=0)$ field, the pressure $\mathrm{P}$ is

$P=\frac{\rho}{3}=\frac{1}{1440 \pi^{2} r^{4}}\left(\frac{4}{3} \pi r^{3}\right) \rho_{v a c}$ 
Therefore,

$P=\frac{2 \gamma^{\prime}}{r}$

Equation 21 agrees with the Young-Laplace equation used to determine the pressure due to surface tension. 


\section{Discussion}

Another possibility for the surface tension has been explored previously. Witten has conjectured that in a first-order QCD phase transition the quark matter may get concentrated in invisible quark nuggets ${ }^{7}$. Further, using a Fermi gas model, Farhi and Jaffe have found the conditions under which quark matter is stable and may not get evaporated in the early universe $^{8}$. Furthermore, Berger conjectured that the quark matter may be the dark matter that closes the universe ${ }^{9}$. However, Alcock and Farhi found that only the strange matter lumps with baryon number larger than $10^{52}$ could survive the evaporation ${ }^{10}$. In contrast, by taking into account the reabsorption of emitted hadrons, Madsen, Heiselberg, and Riisagar ${ }^{11}$ suggest that quark matter with a much lower baryon number could have survived the evaporation. Finally, Berger ${ }^{9}$ calculated the surface tension of quark matter and concluded that it is insufficient to save it from boiling away in the early universe. Thus, quark matter may not be the dark matter that is responsible for the surface tension of the universe. On the other hand, the surface tension of the quark matter may be responsible for the formation of stellar objects such as quark stars ${ }^{12}$ and may have other astrophysical implications ${ }^{13-17}$. Further, the surface tension force originates from the quantum vacuum field because the Young-Laplace equation of the surface tension force is identical to the functional form of the force due to the field. 


\section{Conflict of Interest}

The author declares that he has no conflict of interest. 


\section{References}

1 Cooperstock, F. I. \& Israelit, M. The Energy of the Universe. Found Phys 25, 631635, doi:Doi 10.1007/Bf02059009 (1995).

2 Dymnikova, I. \& Fil'chenkov, M. Quantum birth of a hot universe. Phys Lett B 545, $214-220(2002)$.

3 Ford, L. H. Quantum Vacuum Energy in a Closed Universe. Phys Rev D 14, 33043313, doi:DOI 10.1103/PhysRevD.14.3304 (1976).

4 Kubotani, H., Uesugi, T., Morikawa, M. \& Sugamoto, A. Classification of quantum fluctuation in inflationary universe. Prog Theor Phys 98, 1063-1079 (1997).

5 Nambu, Y. Entanglement of quantum fluctuations in the inflationary universe. Phys Rev D 78 (2008).

6 Sato, K. 1st-Order Phase-Transition of a Vacuum and the Expansion of the Universe. Mon Not R Astron Soc 195, 467-479, doi:DOI 10.1093/mnras/195.3.467 (1981).

7 Witten, E. Cosmic Separation of Phases. Phys Rev D 30, 272-285, doi:DOI 10.1103/PhysRevD.30.272 (1984).

8 Farhi, E. \& Jaffe, R. L. Strange Matter. Phys Rev D 30, 2379-2390, doi:DOI 10.1103/PhysRevD.30.2379 (1984).

9 Berger, M. S. Surface-Tension of Strange-Quark Matter in the Early Universe. Phys Rev D 40, 2128-2130, doi:DOI 10.1103/PhysRevD.40.2128 (1989).

10 Alcock, C. \& Farhi, E. Evaporation of Strange Matter in the Early Universe. Phys Rev D 32, 1273-1279, doi:DOI 10.1103/PhysRevD.32.1273 (1985).

11 Madsen, J., Heiselberg, H. \& Riisager, K. Does Strange Matter Evaporate in the Early Universe. Phys Rev D 34, 2947-2955 (1986).

12 Pinto, M. B., Koch, V.\& Randrup, J. Surface tension of quark matter in a geometrical approach. Phys Rev C 86, doi:Artn 025203 10.1103/Physrevc.86.025203 (2012). 
13 Lugones, G., Grunfeld, A. G. \& Al Ajmi, M. Surface tension and curvature energy of quark matter in the Nambu-Jona-Lasinio model. Phys Rev C 88, doi:Artn 045803 10.1103/Physrevc.88.045803 (2013).

14 Berezhiani, Z., Bombaci, I., Drago, A., Frontera, F. \& Lavagno, A. Gamma-ray bursts from delayed collapse of neutron stars to quark matter stars. Astrophys $J \mathbf{5 8 6}, 1250$ 1253, doi:Doi 10.1086/367756 (2003).

15 Garcia, A. F. \& Pinto, M. B. Surface tension of magnetized quark matter. Phys Rev C 88, doi:Artn 025207 10.1103/Physrevc.88.025207 (2013).

16 Heiselberg, H., Pethick, C. J. \& Staubo, E. F. Quark Matter Droplets in Neutron-Stars. Phys Rev Lett 70, 1355-1359 (1993).

17 Mintz, B. W., Fraga, E. S., Pagliara, G. \& Schaffner-Bielich, J. Nucleation of quark matter in protoneutron star matter. Phys Rev D 81 (2010). 